\title{
Experimental control of the transition from Markovian to non-Markovian dynamics of open quantum systems
}

\author{
Bi-Heng Liu', Li Li ${ }^{1}$, Yun-Feng Huang ${ }^{1}$, Chuan-Feng Li ${ }^{1 \star}$, Guang-Can Guo ${ }^{1}$, Elsi-Mari Laine ${ }^{2}$, \\ Heinz-Peter Breuer ${ }^{3}$ and Jyrki Piilo2ћ
}

\begin{abstract}
Realistic quantum mechanical systems are always exposed to an external environment. This often induces Markovian processes in which the system loses information to its surroundings. However, many quantum systems exhibit nonMarkovian behaviour with a flow of information from the environment back to the system ${ }^{1-5}$. The environment usually consists of large number of degrees of freedom which are difficult to control, but some sophisticated schemes for reservoir engineering have been developed ${ }^{6}$. The control of open systems plays a decisive role, for example, in proposals for entanglement generation ${ }^{7-9}$ and dissipative quantum computation ${ }^{10}$, for the design of quantum memories ${ }^{11}$ and in quantum metrology ${ }^{12}$. Here we report an all-optical experiment which allows one to drive the open system from the Markovian to the non-Markovian regime, to control the information flow between the system and the environment, and to determine the degree of non-Markovianity by measurements on the open system.
\end{abstract}

The standard approach to the dynamics of open quantum systems employs the concept of a quantum Markov process which is given by a semigroup of completely positive dynamical maps and a corresponding quantum master equation with a generator in Lindblad form ${ }^{13,14}$. Very recently, a toolbox for the engineering of such quantum Markov processes in a multi-qubit system of trapped ions has been realized experimentally ${ }^{15}$ and technological developments have also allowed experimental studies of quantum correlations in open systems ${ }^{16,17}$. Within a microscopic approach, quantum Markovian master equations are usually obtained by means of the Born-Markov approximation, which presupposes a weak system-environment coupling and several further, mostly rather drastic approximations. However, in many processes occurring in nature these approximations are not applicable, a situation which occurs, in particular, in the cases of strong systemenvironment couplings, structured and finite reservoirs, and low temperatures, as well as in the presence of large initial systemenvironment correlations. In the case of substantial quantitative and qualitative deviations from the dynamics of a quantum Markov process one often speaks of a non-Markovian process, implying that the dynamics is governed by significant memory effects. Quite recently important steps towards the development of a general consistent theory of non-Markovian quantum dynamics have been made which try to rigorously define the border between Markovian and non-Markovian quantum evolution and to quantify memory effects in the open system dynamics ${ }^{18-21}$.

The measure for quantum non-Markovianity constructed in ref. 19 is based on the idea that memory effects in the open system dynamics can be characterized in terms of the flow of information between the open system and its environment. It has been used recently, for example, to describe this information flow in the energy transfer dynamics of photosynthetic complexes ${ }^{2,4}$, and to characterize memory effects of the dynamics of qubits in spin baths ${ }^{5}$. Here, we present the results of an experiment which enables one, through a careful preparation of the initial systemenvironment states and quantum state tomography, not only to control and to monitor the transition from Markovian to nonMarkovian quantum dynamics, but also the direct determination of this measure for quantum non-Markovianity.

Quantum memory effects are quantified by employing the trace distance $D\left(\rho_{1}, \rho_{2}\right)=(1 / 2) \operatorname{tr}\left|\rho_{1}-\rho_{2}\right|$ between two quantum states $\rho_{1}$ and $\rho_{2}$. This quantity can be interpreted as a measure for the distinguishability of the two states ${ }^{22-24}$. Markovian processes tend to continuously reduce the distinguishability of physical states, which means that there is a flow of information from the open system to its environment. In view of this interpretation the characteristic feature of a non-Markovian quantum process is the increase of the distinguishability, that is a reversed flow of information from the environment back to the open system. Through this recycling of information the earlier states of the open system influence its later states, which expresses the emergence of a memory effect in the open system's dynamics ${ }^{19,20}$.

On the basis of this physical picture one can construct a general measure for the degree of non-Markovianity of a quantum process given by some completely positive dynamical map $\Phi_{t}$ that maps the initial states $\rho(0)$ of the open system to the corresponding states $\rho(t)=\Phi_{t}(\rho(0))$ at time $t$. The full time evolution of the open system over some time interval from the initial time 0 to the final time $T$ is then given by a one-parameter family $\Phi=\left\{\Phi_{t} \mid 0 \leq t \leq T\right\}$ of dynamical maps. Completely positive maps are contractions for the trace distance. Hence, considering two different initial states $\rho_{1}(0)$ and $\rho_{2}(0)$, with corresponding time evolutions $\rho_{1}(t)=\Phi_{t}\left(\rho_{1}(0)\right)$ and $\rho_{2}(t)=\Phi_{t}\left(\rho_{2}(0)\right)$, the trace distance $D\left(\rho_{1}(t), \rho_{2}(t)\right)$ at time $t>0$ can never be larger than the trace distance $D\left(\rho_{1}(0), \rho_{2}(0)\right)$ at the initial time $t=0$. However, this contraction property does not imply that $D\left(\rho_{1}(t), \rho_{2}(t)\right)$ is

\footnotetext{
${ }^{1}$ Key Laboratory of Quantum Information, University of Science and Technology of China, CAS, Hefei 230026, China, ${ }^{2}$ Turku Centre for Quantum Physics, Department of Physics and Astronomy, University of Turku, FI-20014 Turun yliopisto, Finland, ${ }^{3}$ Physikalisches Institut, Universität Freiburg, Hermann-Herder-Strasse 3, D-79104 Freiburg, Germany. *e-mail: cfli@ustc.edu.cn; jyrki.piilo@utu.fi.
} 


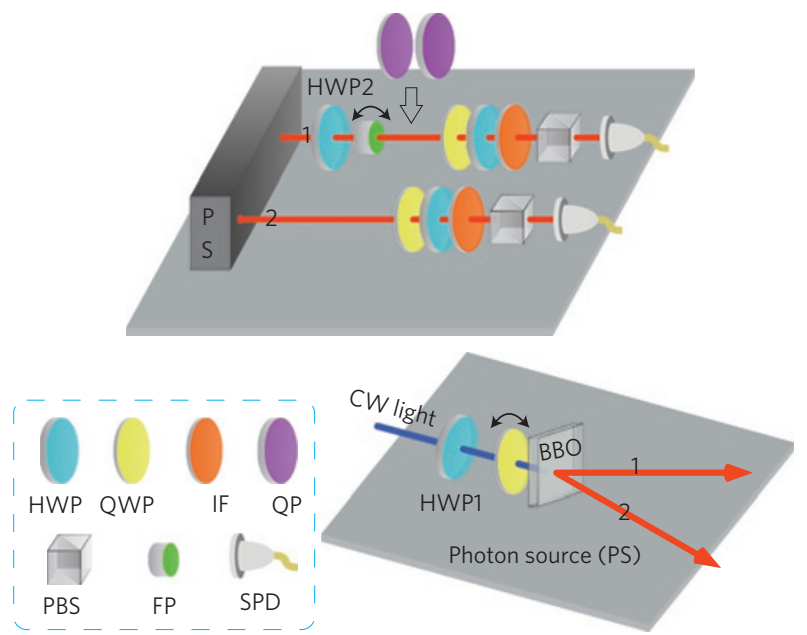

Figure 1 | The experimental set-up. Key to the components: HWP-half-wave plate, QWP-quarter-wave plate, IF-interference filter, QP-quartz plate, PBS - polarizing beamsplitter, FP-Fabry-Pérot cavity, and SPD-single photon detector.

a monotonically decreasing function of time, and in fact any temporary increase of the trace distance is a signature of quantum memory effects. Defining the rate of change of the trace distance by $\sigma\left(t, \rho_{1,2}(0)\right)=(\mathrm{d} / \mathrm{d} t) D\left(\rho_{1}(t), \rho_{2}(t)\right)$, our measure $\mathcal{N}(\Phi)$ for the non-Markovianity of the process is therefore given by

$$
\mathcal{N}(\Phi)=\max _{\rho_{1,2}(0)} \int_{\sigma>0} \mathrm{~d} t \sigma\left(t, \rho_{1,2}(0)\right)
$$

Here, the time-integration is extended over all subintervals of $[0, T]$ in which the rate of change of the trace distance $\sigma$ is positive, and the maximum is taken over all pairs of initial states. The quantity (1) thus measures the maximal total increase of the distinguishability over the whole time-evolution, that is, the maximal total amount of information that flows from the environment back to the open system.

In our experiment the open quantum system is provided by the polarization degree of freedom of photons coupled to the frequency degree of freedom representing the environment. The experimental set-up is shown in Fig. 1. An ultraviolet argon-ion laser is used to pump two $0.3 \mathrm{~mm}$ thick $\mathrm{BBO}$ crystals cut for a type I downconversion process to generate arbitrary pure two-qubit states. A fused silica plate $(0.04 \mathrm{~mm}$ thick and coated with a partial reflecting coating on each side, with approximately $85 \%$ reflectivity at $702 \mathrm{~nm}$ ) is used as a Fabry-Pérot (FP) cavity. The cavity is mounted on a rotator which can be tilted in the horizontal plane. A $4 \mathrm{~nm}$ (full width at half maximum) interference filter is placed after the FP cavity to filter out at most two transmission peaks. The corresponding interference filter in the other arm is $10 \mathrm{~nm}$. The polarization and frequency degrees of freedom are coupled in a quartz plate in which different evolution times are realized by varying the thickness of the plate. A polarizing beam splitter together with a half-wave plate and a quarter-wave plate is used as a photon state analyser.

The half-wave plate HWP2 and the tilted FP cavity are used to prepare the initial one-photon states $\left|\psi_{1,2}(0)\right\rangle=\left|\varphi_{1,2}\right\rangle \otimes|\chi\rangle$, where

$$
\left|\varphi_{1,2}\right\rangle=\frac{1}{\sqrt{2}}(|H\rangle \pm|V\rangle)
$$

with $|H\rangle$ and $|V\rangle$ denoting the horizontal and the vertical polarization states, respectively. The environmental state $|\chi\rangle=\int \mathrm{d} \omega f(\omega)|\omega\rangle$ involves the amplitude $f(\omega)$ for the photon to be in a mode with frequency $\omega$, which is normalized as

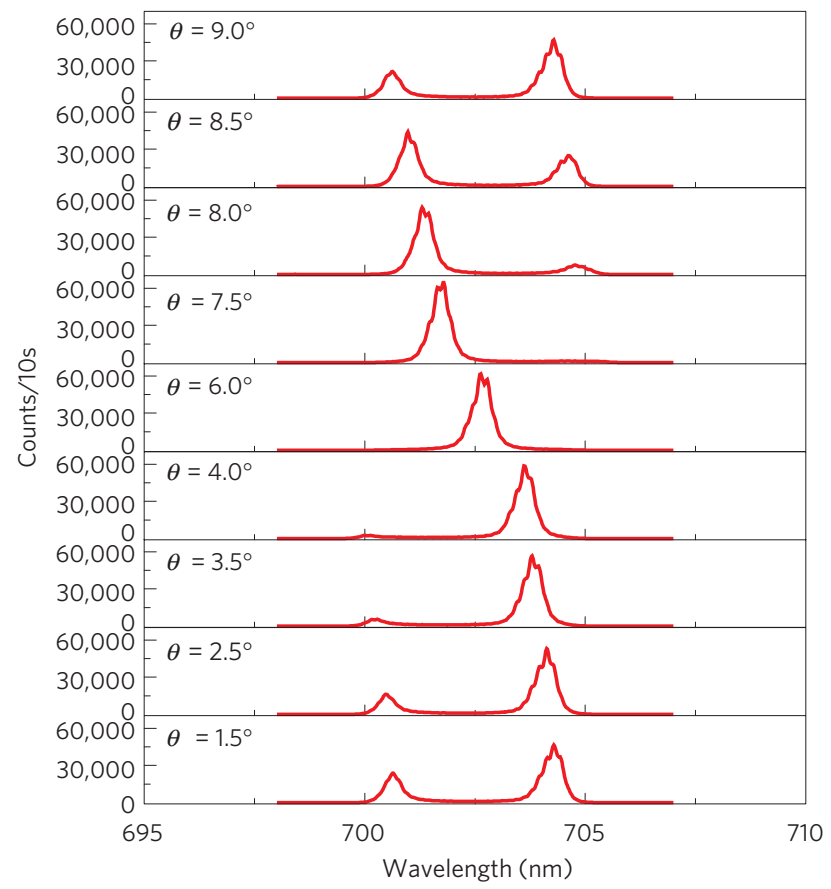

Figure 2 | The frequency spectrum of the initial state for various values of the tilt angle $\boldsymbol{\theta}$. See the Supplementary Information for further details.

$\int \mathrm{d} \omega|f(\omega)|^{2}=1$. The form of the frequency distribution $|f(\omega)|^{2}$, and thus the initial state of the environment, can be controlled by the tilt angle $\theta$ of the FP cavity. Figure 2 shows how $\theta$ determines the structure of the frequency spectrum and, thus, the environmental initial state $|\chi\rangle$. By changing the initial state of the environment in the experiment we modify the open system dynamics in a way which allows us to observe transitions between Markovian and non-Markovian quantum dynamics.

In the first version of the experiment, HWP1 is fixed at zero degrees to generate a two-photon state. Photon 2 is directly detected in detector SPD at the end of arm 2 as a trigger for photon 1. Photon 1 first passes through HWP2, preparing it in the state $\left|\varphi_{1}\right\rangle$ or $\left|\varphi_{2}\right\rangle$. The subsequent interaction between polarization and mode degrees of freedom in the quartz plate is described by a quantum dynamical map $\Phi_{t}$ acting on the open system, where the interaction time $t$ is related to the variable length $L$ of the quartz plate as $t=L / c$. Finally, full state tomography is carried out in detector SPD at the end of arm 1 to determine the polarization state $\rho_{1,2}(t)=\Phi_{t}\left(\left|\varphi_{1,2}\right\rangle\left\langle\varphi_{1,2}\right|\right)$ of photon 1 . This allows the direct experimental determination of the trace distance $D\left(\rho_{1}(t), \rho_{2}(t)\right)$ between the two possible one-photon states after a certain interaction time $t$, controlled by the length $L$ of the quartz plate. Experimental results for four different values of the tilt angle $\theta$ of the FP cavity are shown in Fig. 3a. We clearly observe a crossover from a monotonic to a non-monotonic behaviour of the trace distance as a function of time, that is, a transition from a Markovian to a non-Markovian dynamics for the polarization degree of freedom of the photon.

The experimental results admit a simple theoretical analysis which is based on the fact that the time evolution in the quartz plate may be described by the unitary operator $U(t)$, which is defined by

$$
U(t)|\lambda\rangle \otimes|\omega\rangle=\mathrm{e}^{i n_{\lambda} \omega t}|\lambda\rangle \otimes|\omega\rangle
$$

where $n_{\lambda}$ represents the refraction index for light with polarization $\lambda=H, V$. The presence of the quartz plate thus leads to the decoherence of superpositions of polarization states, which is due to a nonzero difference $\Delta n=n_{\mathrm{V}}-n_{\mathrm{H}}$ in the refraction indices of 
a

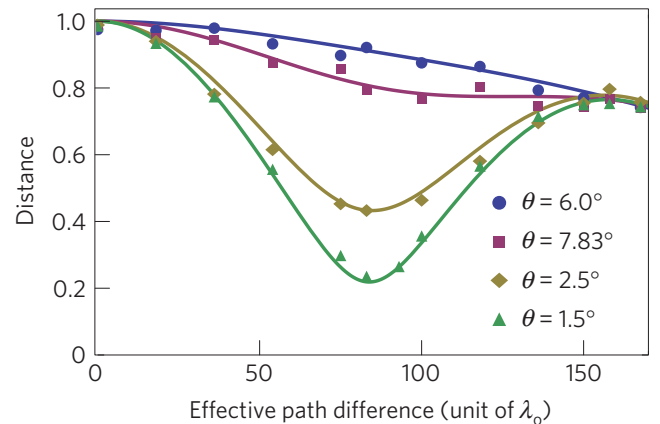

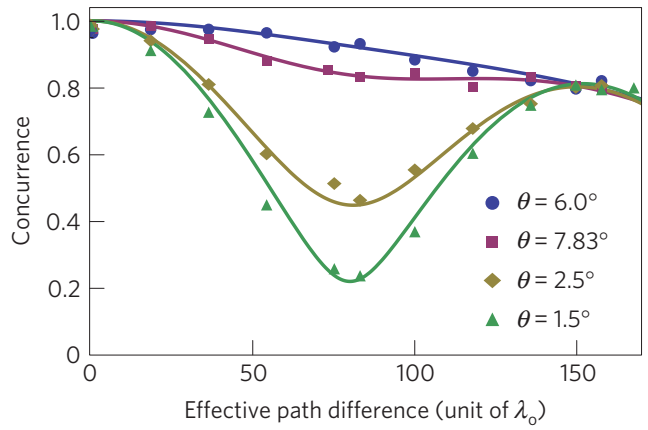

Figure 3 | The distance and the concurrence as a function of the effective path difference for four different values of the tilt angle $\boldsymbol{\theta}$. a, The trace distance. b. The concurrence between the system and the ancilla. The solid lines represent the theoretical predictions ( 3 ) for $\sigma=1.8 \times 10^{12} \mathrm{~Hz}$ (corresponding to $0.47 \mathrm{~nm}$ ) and $\Delta \omega=1.6 \times 10^{13} \mathrm{~Hz}$ (corresponding to $4.19 \mathrm{~nm}$ ). The effective path difference is equal to $\Delta \mathrm{nL}$ and $\lambda_{0}=702 \mathrm{~nm}$. The experimental error bars due to the counting statistics are smaller than the symbols. Further experimental details may be found in the Supplementary Information.

horizontally and vertically polarized photons. The corresponding dynamical map $\Phi_{t}$ takes the form:

$$
\Phi_{t}:\left\{\begin{aligned}
&|H\rangle\langle H| \mapsto|H\rangle\langle H|, \\
&|V\rangle\langle V|\mapsto| V\rangle\langle V|, \\
&|H\rangle\left\langle V\left|\mapsto \kappa^{*}(t)\right| H\right\rangle\langle V|, \\
&|V\rangle\langle H|\mapsto \kappa(t)| V\rangle\langle H|
\end{aligned}\right.
$$

where the decoherence function $\kappa(t)$ is given by the Fourier transform of the frequency distribution

$$
\kappa(t)=\int \mathrm{d} \omega|f(\omega)|^{2} \mathrm{e}^{\mathrm{i} \omega \Delta n t}
$$

With the help of these relations it is easy to show that the trace distance corresponding to the initial pair of states (2) is equal to the modulus of the decoherence function

$$
D\left(\rho_{1}(t), \rho_{2}(t)\right)=|\kappa(t)|
$$

In the experiment the transition from Markovian to nonMarkovian dynamics is observed through variation of the tilt angle $\theta$ of the FP cavity. As illustrated in Fig. 2, all frequency distributions are very well approximated by a sum of two Gaussians centred at frequencies $\omega_{k}$, with amplitudes $A_{k}$ and equal widths $\sigma$, which yields

$$
|\kappa(t)|=\frac{\mathrm{e}^{-\frac{1}{2} \sigma^{2}(\Delta n t)^{2}}}{1+A_{\theta}} \sqrt{1+A_{\theta}^{2}+2 A_{\theta} \cos (\Delta \omega \cdot \Delta n t)}
$$

where $A_{1}=1 /\left(1+A_{\theta}\right), A_{2}=A_{\theta} /\left(1+A_{\theta}\right)$ and $\Delta \omega=\omega_{2}-\omega_{1}$. As can be seen from the frequency spectra of Fig. 2, the relative amplitude $A_{\theta}$ varies strongly with the tilt angle $\theta$ and represents the relevant parameter controlling the transition, whereas the widths of the peaks, $\sigma$, and the distance between them, $\Delta \omega$, are almost constant. To fit the experimental data shown in Fig. 3 to the theoretical prediction (3) we use $A_{\theta}$ as the fit parameter, taking $\Delta \omega=1.6 \times 10^{13} \mathrm{~Hz}$ and $\sigma=1.8 \times 10^{12} \mathrm{~Hz}$ as fixed. The results are shown as continuous curves in Fig. 3, demonstrating an excellent agreement with the experimental data.

The experiment also enables a direct determination of the measure for non-Markovianity (1). First, we note that the initial pair (2) is already optimal in the sense that it yields a maximal increase of the trace distance. The theoretical explanation for this fact is presented in the Supplementary Information. Second, in our experiment the increase of the trace distance is restricted to a single

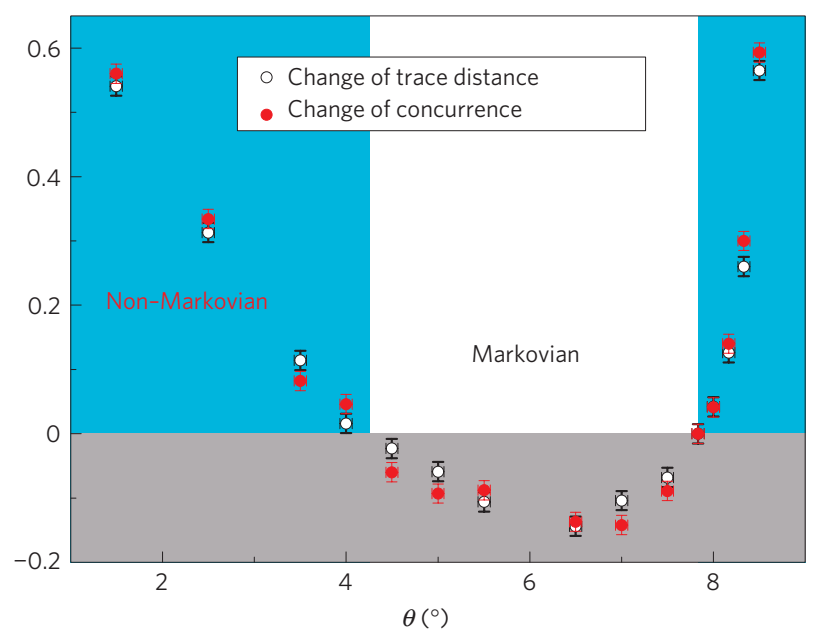

Figure 4 | The change of the trace distance and of the concurrence as functions of the tilt angle $\boldsymbol{\theta}$. The transition from the non-Markovian to the Markovian regime occurs at $\theta \simeq 4.1^{\circ}$, and from the Markovian to the non-Markovian regime at $\theta \simeq 8.0^{\circ}$. The positive values in the blue regions directly give the non-Markovianity measure $\mathcal{N}(\Phi)$ of the process. The negative values in the grey area correspond to $\mathcal{N}(\Phi)=0$, that is, to Markovian dynamics. The error bars are due to the uncertainty of the tilt angle and the counting statistics.

time interval (see Fig. 3). The non-Markovianity measure (1) is thus obtained by determining the difference of the trace distance between the first local minimum and the subsequent maximum. Our experimental results are shown in Fig. 4. Increasing the tilt angle of the cavity decreases the relative amplitude $A_{\theta}$ between the peaks in the frequency spectrum, and thereby reduces the non-Markovianity of the process until a transition to Markovian dynamics occurs. Further increasing the tilt angle amplifies the relative amplitude again and brings the dynamics back to the non-Markovian regime.

In ref. 21 an alternative measure for non-Markovianity has been proposed that is based on the idea that Markovian dynamics leads to a monotonic decrease of the entanglement between the open system and an isomorphic ancilla system, whereas a non-Markovian dynamics induces a temporary increase of the entanglement. One can show (see Supplementary Information) that for the present experiment this measure coincides with (1) if one uses the concurrence ${ }^{25,26}$ as an entanglement measure. This fact leads to an alternative and independent method for the measurement of the non-Markovianity by means of our experimental setup. Fixing HWP1 to $22.5^{\circ}$, we generate a maximally entangled 
two-photon state $(1 / \sqrt{2})(|H H\rangle+|V V\rangle)$. Photon 1 then passes the quartz plate and the composite final state is analysed through two-photon state tomography. Experimental results are shown in Figs $3 \mathrm{~b}$ and 4, clearly demonstrating the equivalence of both measures for non-Markovianity.

Our experiment clearly reveals the measurability of recently proposed theoretical measures for quantum non-Markovianity which yield important information about the type of quantum noise and about environmental properties, even when the environment is a complex system involving an infinite number of degrees of freedom and is not directly accessible through measurements. Moreover, we have introduced a method for the control of the information flow between the open system and its environment, which opens the possibility of efficiently exploiting memory effects in future quantum technologies ${ }^{27}$.

\section{Received 5 May 2011; accepted 8 August 2011; published online 11 September 2011}

\section{References}

1. Breuer, H-P. \& Petruccione, F. The Theory of Open Quantum Systems (Oxford Univ. Press, 2007)

2. Lee, H., Cheng, Y-C. \& Fleming, G. R. Coherence dynamics in photosynthesis: Protein protection of excitonic coherence. Science 316, 1462-1465 (2007).

3. Cederbaum, L. S., Gindensperger, E. \& Burghardt, I. Short-time dynamics through conical intersections in macrosystems. Phys. Rev. Lett. 94, 113003 (2005)

4. Rebentrost, P. \& Aspuru-Guzik, A. Exciton-phonon information flow in the energy transfer process of photosynthetic complexes. J. Chem. Phys. 134, 101103 (2011)

5. Tony, J. G., Apollaro, T. J. G., Di Franco, C., Plastina, F. \& Paternostro, M. Memory-keeping effects and forgetfulness in the dynamics of a qubit coupled to a spin chain. Phys. Rev. A 83, 032103 (2011).

6. Myatt, C. J. et al. Decoherence of quantum superpositions through coupling to engineered reservoirs. Nature 403, 269-273 (2000).

7. Diehl, S. et al. Quantum states and phases in driven open quantum systems with cold atoms. Nature Phys. 4, 878-883 (2008).

8. Krauter, H. et al. Entanglement generated by dissipation and steady state entanglement of two macroscopic objects. Phys. Rev. Lett. 107, 080503 (2011).

9. Cho, J., Bose, S. \& Kim, M. S. Optical pumping into many-body entanglement. Phys. Rev. Lett. 106, 020504 (2011).

10. Verstraete, F., Wolf, M. M. \& Cirac, J. I. Quantum computation and quantum-state engineering driven by dissipation. Nature Phys. 5, 633-636 (2009).

11. Pastawski, F., Clemente, L. \& Cirac, J. I. Quantum memories based on engineered dissipation. Phys. Rev. A 83, 012304 (2011).
12. Goldstein, G. et al. Environment assisted precision measurement. Phys. Rev. Lett. 106, 140502 (2011).

13. Gorini, V., Kossakowski, A. \& Sudarshan, E. C. G. Completely positive dynamical semigroups of N-level systems. J. Math. Phys. 17, 821-825 (1976).

14. Lindblad, G. On the generators of quantum dynamical semigroups. Commun. Math. Phys. 48, 119-130 (1976).

15. Barreiro, J. T. et al. An open-system quantum simulator with trapped ions. Nature 470, 486-491 (2011).

16. Xu, J-S. et al. Experimental characterization of entanglement dynamics in noisy channels. Phys. Rev. Lett. 103, 240502 (2009).

17. Xu, J-S. et al. Experimental investigation of classical and quantum correlations under decoherence. Nature Commun. 1, 7 (2010).

18. Wolf, M. M. et al. Assessing non-Markovian quantum dynamics. Phys. Rev. Lett. 101, 150402 (2008).

19. Breuer, H-P., Laine, E-M. \& Piilo, J. Measure for the degree of non-Markovian behavior of quantum processes in open systems. Phys. Rev. Lett. 103, 210401 (2009).

20. Laine, E-M., Piilo, J. \& Breuer, H-P. Measure for the non-Markovianity of quantum processes. Phys. Rev. A 81, 062115 (2010).

21. Rivas, A., Huelga, S. F. \& Plenio, M. B. Entanglement and non-Markovianity of quantum evolutions. Phys. Rev. Lett. 105, 050403 (2010).

22. Helstrom, C. W. Quantum Detection and Estimation Theory (Academic, 1976).

23. Holevo, A. S. An analog of the theory of statistical decisions in noncommutative theory of probability. Trans. Moscow Math. Soc. 26, 133-149 (1972).

24. Hayashi, M. Quantum Information (Springer, 2006).

25. Wootters, W. K. Entanglement of formation of an arbitrary state of two qubits. Phys. Rev. Lett. 80, 2245-2248 (1998).

26. Rungta, P. et al. Universal state inversion and concurrence in arbitrary dimensions. Phys. Rev. A 64, 042315 (2001).

27. Perdomo, A. et al. Engineering directed excitonic energy transfer. Appl. Phys. Lett. 96, 093114 (2010).

\section{Acknowledgements}

This work was supported by the National Fundamental Research Program, National Natural Science Foundation of China (Grant Nos. 60921091, 10874162 and 10734060), the Magnus Ehrnrooth Foundation, and the Graduate School of Modern Optics and Photonics.

\section{Author contributions}

B-H.L., L.L., Y-F.H., C-F.L. and G-C.G. planned, designed and implemented the experiments. E-M.L., H-P.B. and J.P. carried out the theoretical analysis and developed the interpretation. B-H.L., C-F.L., E-M.L, H-P.B. and J.P. wrote the paper and all authors discussed its contents.

\section{Additional information}

The authors declare no competing financial interests. Supplementary information accompanies this paper on www.nature.com/naturephysics. Reprints and permissions information is available online at http://www.nature.com/reprints. Correspondence and requests for materials should be addressed to C-F.L. or J.P. 